\title{
Radio Frequency Response of Magnetic Nanoparticle-Doped Yarn
}

\author{
B. Dolník ${ }^{a, *}$, M. RajŇÁK ${ }^{a, b}$, R. Cimbala $^{a}$, I. Kolcunová ${ }^{a}$, J. KurimskÝ ${ }^{a}$, J. DŽmura ${ }^{a}$, \\ J. Petrás $\check{S}^{a}$, J. ZBojovskÝ ${ }^{a}$, J. UrbanskÝ ${ }^{a}$, Z. Mitróová ${ }^{b}$, J. VAlterA ${ }^{c}$ And T. Kalous ${ }^{d}$ \\ ${ }^{a}$ Faculty of Electrical Engineering and Informatics, Department of Electrical Power Engineering, \\ Technical University of Košice, Mäsiarska 74, 04120 Košice, Slovakia \\ ${ }^{b}$ Institute of Experimental Physics, SAS, Watsonova 47, 04001 Košice, Slovakia \\ ${ }^{c}$ Faculty of Mechanical Engineering, Department of Textile Machine Design, \\ Technical University of Liberec, Liberec, 46117, Czech Republic \\ ${ }^{d}$ Faculty of Textile Engineering, Department of Nonwovens and Nanofibrous Materials, \\ Technical University of Liberec, Liberec, 46117, Czech Republic
}

\begin{abstract}
The amount of research with electrospun nanofibers in various fields of science and their use in engineering applications is increasing rapidly. The commercial use of nanofibre technology is intensively investigated mainly in the field of water treatment and environmental remediation, health and biomedical engineering, and energy production and storage. Therefore, a wide range of base materials has been used to synthesize nanofibers with various physical properties. This paper presents the results of experiments on the shielding effectiveness against electromagnetic fields using yarns. The yarns were produced using AC electrospinning based on a needleless spinning-electrode. The optimal frequency of $50 \mathrm{~Hz}$ and a high voltage of $40 \mathrm{kV}$ were used to generate the yarn. The poly(vinylbutyral) was chosen as a polymer. Nanoparticles of $\mathrm{Fe}_{3} \mathrm{O}_{4}$ were used as the filler. Transmission electron microscopy confirmed good compatibility of the nanoparticles with polymeric nanofibers. The presence of the magnetic dopant in the nanofibers was also reflected in measured magnetization curves. Measurements of $\mathrm{Fe}_{3} \mathrm{O}_{4}$ nanoparticle-doped yarn shielding effectiveness in the frequency range from $700 \mathrm{MHz}$ to $3 \mathrm{GHz}$ confirmed a slight shielding effect.
\end{abstract}

DOI: 10.12693/APhysPolA.137.687

PACS/topics: yarn, nanoparticle, radio frequency, shielding effectiveness

\section{Introduction}

Among the techniques used in nanotechnology, electrospinning is becoming a rather important technology because of its ability to generate nanostructures with unique properties. Since the beginning of the 20th century, when electrospinning began to be used, there have been significant improvements in tool design, materials used (nanoparticles), and manufactured nanomaterials [1].

Electrospun nanofibers play a key role in the field of biomaterials, where their use in biomedical engineering is anticipated using biocompatible and biodegradable polymers as drug carriers to target sites in living organisms $[2,3]$. Electrospun nanofibers are used in applications such as large surface sensors [4-6], nanotubes [7], catalysers [4, 8], tissue engineering [9], and electronics [4]. Last but not least, the environmental applications of nanofibers should be mentioned, where they are used as separator with selective oil/water affinity [4].

One area of interest that has not yet been explored by the scientific community is the interaction of magnetic

*corresponding author; e-mail: bystrik.dolnik@tuke.sk nanoparticle-doped yarn to radio frequency electromagnetic field, namely its shielding effectiveness (SE). Here we present the results obtained by experimental measurements of the SE of self-made magnetic nanoparticledoped yarn (MNPDY) sample using AC electrospinning based on a needleless spinning-electrode.

\section{Theoretical background}

Measuring SE for newly developed materials is crucial to determine their properties and potential applications. The radio frequency $(\mathrm{RF})$ response is one of the main indicators for measuring the SE. One of the common technique for testing SE that can be found anywhere in the electromagnetic compatibility (EMC) field [10] is the open field test. The SE of a material investigated is defined as follows [11]:

$\mathrm{SE}_{\mathrm{dB}}=10 \log \left(\frac{P_{i}}{P_{t}}\right)=20 \log \left(\frac{E_{i}}{E_{t}}\right)=20 \log \left(\frac{H_{i}}{H_{i}}\right)$.

In Eq. (1) the $P_{i}, E_{i}$, and $H_{i}$ are the RF power, the electric and magnetic field intensity incident on the barrier (shield) and $P_{t}, E_{t}$, and $H_{t}$ are the counterparts transmitted through the barrier. When the instrument readings $V_{t}$ and $V_{i}$ are, respectively, proportional to $E_{t}$ and $E_{i}\left(H_{t}\right.$ and $\left.H_{i}\right)$, as is the usual measurement situation, an another form for (1) is as follows:

$$
\mathrm{SE}_{\mathrm{dB}}=V_{i(\mathrm{~dB})}-V_{t(\mathrm{~dB})} \text {. }
$$


When the distance from the EM wave source to shield material is less than $\frac{\lambda}{2 \pi} \approx \frac{\lambda}{6}$ of the free path wavelength of the EM wave to be shielded, the radiation is described as a near field region. The region above this is in the far field region.

\section{Materials and methods}

The magnetic nanocomposite was based on the mixture of mineral oil-based magnetic nanofluid (MNF) and polyvinylbutyral (PVB), since this prototypical polymer can be used for electrospinning at high voltages [12]. A $10 \mathrm{wt} \%$ solution of PVB was prepared in ethanolisopropyl alcohol (4:1). This solution $(45 \mathrm{ml})$ was mixed at room temperature with $4 \mathrm{ml}$ of the magnetic nanofluid containing 6.6 vol. $\%$ of iron oxide nanoparticles $\left(\mathrm{Fe}_{3} \mathrm{O}_{4}\right)$ with the mean particle diameter of $10 \mathrm{~nm}$ [13]. Thus, the resulting mixture of $10 \mathrm{wt} \% \mathrm{PVB}$ and MNF contained 0.54 vol. $\%$ of nanoparticles. The prepared magnetic colloid was then electrospun following the well-known electrospinning approach [1]. For electrospinning we used an AC high voltage of $40 \mathrm{kV}$ with power frequency of $50 \mathrm{~Hz}$, which was optimal for spinning of the used solution.

Magnetization of the MNPDY was measured by means of a vibrating sample magnetometer (VSM) installed on a cryogen free superconducting magnet from Cryogenic Ltd. The measurements were performed at room temperature and magnetic field up to 5 T. Delong LVEM5 low voltage transmission electron microscope was used to obtain the microscopic image of the MNPDY.

We used two mutually oriented antennas to study the MNPDY response to the EM RF field. The SE measurement was made at room temperature in the near field region with the predominant magnetic field component. The Agilent N5183A harmonic generator was used to power the transmitting antenna. The received signal level was measured by the R\&S FSH8 spectrum analyzer. The microclimate conditions during the measurements in the anechoic chamber were as follows: ambient temperature $(22 \pm 1){ }^{\circ} \mathrm{C}$ and relative air humidity $(32 \pm 3) \%$. The thickness of the tested MNPDY sample was $(10 \pm 0.5) \mu \mathrm{m}$.

\section{Results and discussion}

In Fig. 1 the transmission electron microscopy (TEM) of the MNPDY prepared sample used in the experiment is depicted. As can be seen from Fig. 1, the TEM on the MNPDY confirmed good compatibility of the nanoparticles (NP) with polymeric nanofibers.

In Fig. 2, the magnetic field dependent magnetization is presented for the raw yarn and the one doped with the magnetic nanoparticles (MNP). It is evident that the magnetization of the raw yarn opposes the increasing magnetic field, thereby exhibiting the diamagnetic behaviour. On the other hand, the pronounced superparamagnetic magnetization curve was obtained for the MNPDY. The related magnetization curve shows zero hysteresis. Clearly, the superparamagnetic behavior

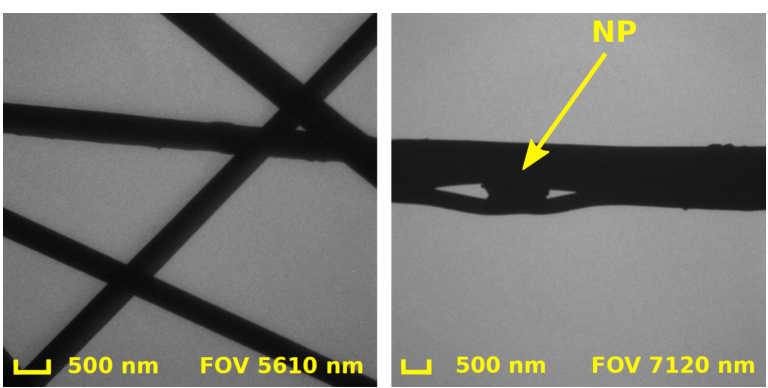

Fig. 1. The TEM of the pure yarn (left) and the MNPDY with one nanoparticle (right) prepared by the electrospinning approach by using an AC high voltage of $40 \mathrm{kV}$.

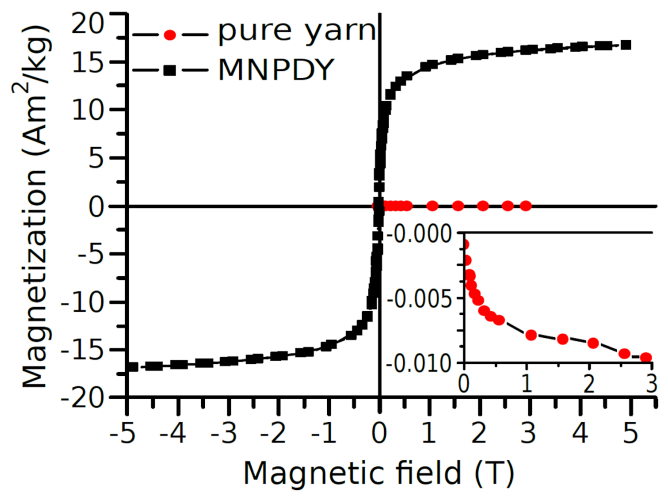

Fig. 2. Magnetization curve measured on the raw yarn and the MNPDY. The inset shows zoomed view on the diamagnetic behavior of the pure yarn.

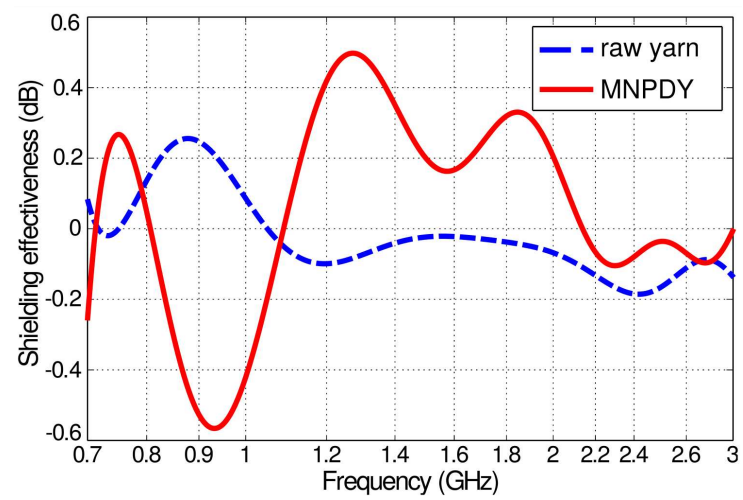

Fig. 3. The calculated SE of the raw yarn (blue dashed curve) and the MNPDY (red curve) investigated in the frequency range from $700 \mathrm{MHz}$ to $3 \mathrm{GHz}$.

stems from the MNP contribution, under which the diamagnetic signal from the yarn is negligible. At $5 \mathrm{~T}$, the magnetization of the MNP in the yarn is well saturated, reaching the value of $16.8 \mathrm{~A} \mathrm{~m}^{2} / \mathrm{kg}$.

By using (2), the MNPDY frequency dependence of the SE within the range studied was calculated. The calculated SE of the MNPDY in the frequency range 
from $750 \mathrm{MHz}$ to $3 \mathrm{GHz}$ is depicted in Fig. 3. As can be seen from the MNPDY SE frequency dependence in Fig. 3, the positive values of the MNPDY SE vary between $0.2 \mathrm{~dB}$ to $0.4 \mathrm{~dB}$ in the frequency range from $1.1 \mathrm{GHz}$ to $2 \mathrm{GHz}$. The narrower SE band is distributed between $710 \mathrm{MHz}$ and $800 \mathrm{MHz}$ with a maximum SE value of $0.27 \mathrm{~dB}$. A negative value of $\mathrm{SE}$ for certain frequencies indicates the presence of the multi-reflection component $M_{(\mathrm{dB})}$ due to the presence of large surface areas (e.g., a porous or foam material) or interface areas in the shield [11]. For comparison in Fig. 3, we present the calculated SE of the raw yarns.

\section{Conclusions}

Measurements of the SE of the MNPDY in the RF range were conducted. The results obtained by measuring the SE in the near field region pointed to the positive values of the $\mathrm{SE}$ between $0.2 \mathrm{~dB}$ to $0.4 \mathrm{~dB}$ in the frequency range from $1.1 \mathrm{GHz}$ to $2 \mathrm{GHz}$. The measured SE confirmed a slight shielding effect of the doped yarn, while the raw yarn does not have shielding effects. We suppose that a negative value of SE for certain frequencies is due to the multi-reflection component $M_{(\mathrm{dB})}$ which is typical for materials with a large surface area.

\section{Acknowledgments}

This work was supported by the Scientific Grant Agency of the Ministry of Education of the Slovak Republic and the Slovak Academy of Sciences under the project VEGA No. 2/0141/16, No. 1/0435/19, and No. 1/0340/18; the Cultural and Educational Grant
Agency of the Ministry of Education, Science, Research and Sport of the Slovak Republic under the project No. 008TUKE-4/2019; the Slovak Research and Development Agency under the contract No. APVV-18-0160.

\section{References}

[1] A. Haider, S. Haider, I.K. Kang, Arab. J. Chem. 11, (2018).

[2] G. Wei, P.X. Ma, Adv. Function. Mater. 18, (2008).

[3] A. Haider, K.Ch. Gupta, I.K. Kang, BioMed Res. Int. 2014, (2014).

[4] Kenry, Chwee Teck Lim, Prog. Polym. Sci. 70, 1 (2017).

[5] X. Wang, Ch. Drew, S.H. Lee, K.J. Senecal, J. Kumar, L.A. Samuelson, Nano Lett. 2, (2002).

[6] B. Ding, M. Wang, X. Wang, J. Yu, G. Sun, Mater. Today 13, (2010).

[7] M.M. Hohman, M. Shin, G. Rutledge, M.P. Brenner, Phys. Fluids 13, (2001).

[8] H. Katepalli, M. Bikshapathi, Ch.S. Sharma, N. Verma, A. Sharma, Chem. Eng. J. 171, (2011).

[9] M. Moffa, A. Polini, A.G. Sciancalepore, L. Persano, E. Mele, L.G. Passione, G. Potentecd, D. Pisignano, Soft Matter 9, (2013).

[10] J. Hallon, K. Kováč, Proced. Eng. 69, (2014).

[11] Ch.S. Zhang, Q.Q. Ni, S.Y. Fu, K. Kurashiki, Compos. Sci. Technol. 67, (2007).

[12] P. Pokorny, E. Kostakova, F. Sanetrnik, et al., Phys. Chem. Chem. Phys. 16, (2014).

[13] M. Rajňák, Z. Špitalský, B. Dolník, J. Kurimský, L. Tomčo, R. Cimbala, P. Kopčanský, M. Timko, Phys. Rev. Appl. 11, (2019). 